\title{
LA DIMENSIÓN INTERSUBJETIVA DE LA EXISTENCIA EN LA FILOSOFÍA DE GABRIEL MARCEL
}

\author{
MARTÍN GRASSI \\ Universidad Católica Argentina
}

\begin{abstract}
RESUMEN: El presente trabajo intenta introducir al lector en las reflexiones en torno a la intersubjetividad como dimensión esencial de la existencia personal según Gabriel Marcel. Para ello, se abordará primero qué entiende Marcel por persona; segundo, cómo se comprenden las palabras yo y otro en las relaciones intersubjetivas concretas; por último, se profundizará en el modo en que el otro como otro me es dado, a partir de la noción de presencia. El objetivo principal de este artículo es el de mostrar que la existencia personal es incomprensible sin la referencia a un otro, es decir, que la mismidad no tiene ningún sentido sin la alteridad. Pero esta intencionalidad esencial del sí mismo al otro, no comporta un carácter práctico o accidental, sino que adquiere en Marcel un carácter metafísico, en tanto que la comunión constituye el ser mismo de lo personal.
\end{abstract}

PALABRAS CLAVE: Marcel, intersubjetividad, persona, alteridad, presencia, comunión.

\section{Intersubjective Dimension of Existence in the Philosophy of Gabriel Marcel}

ABTRACT: The present work intends to introduce the reader to the essential dimension of intersubjectivity in personal existence in Gabriel Marcel's philosophy. We will first attend to the characterization of persona; secondly, how we should understand the terms / and the other in concrete interpersonal relationships; in the third place, we will analize the way in which the other is given as an other, through the notion of presence. The main objective of this article is to show that personal existence is uncomprehensible without its reference to the other, that is to say, that identity has no sense without alterity. Moreover, the essential intencionality of identity to alterity does not imply only a practical or accidental character, but a metaphysical one, as communion constitutes the very being of personal existence.

KEY WORDS: Marcel, intersubjectivity, persona, alterity, presence, communion.

\section{La Persona}

La filosofía concreta de Marcel se define ante todo por la centralidad de la existencia frente a las exigencias de un pensamiento objetivista ${ }^{1}$. Si bien la existencia se encuentra definida por su dimensión encarnada y temporal, es preciso interrogar esas dimensiones en orden a explicitar la puesta entre paréntesis de la alteridad a la que está también referido necesariamente el existente ${ }^{2}$. Sin embargo, ya en estas consideraciones

\footnotetext{
1 Sobre la «filosofía concreta» de Gabriel Marcel, cf.: Grassi, M. «La reflexión segunda y el acceso al misterio del Ser en la filosofía concreta de Gabriel Marcel», en: Nuevo Pensamiento: Revista de Filosofía. Universidad del Salvador (Área San Miguel), Vol. 1, Año 1, 2011, pp. 21-46 ( http://mabs.com. ar/nuevopensamiento/index.php/nuevopensamiento/index ). También: Grassi, M. «La filosofía concreta de Gabriel Marcel». En: PERSONA. Revista Iberoamericana de Personalismo Comunitario, N ${ }^{\circ} 17$, año VI, Agosto 2011, pp. 13-18 (http://www.personalismo.net/persona/).

2 Cf. Grassi, M. «El hombre como ser encarnado y la filosofía concreta de Gabriel Marcel». En: Revista de Humanidades (Chile: Universidad Andrés Bello), Vol. 19-20, 2009 (junio-diciembre), pp. 9-28. También: Grassi, M. «La significación ontológico-existencial del tiempo en la filosofía de Gabriel Marcel», en: Revista Philosophia, Facultad de Filosofía y Letras, Universidad Nacional de Cuyo, 73/2 (2013),
} 
respecto al cuerpo propio, al tiempo vivido, y a la exigencia ontológica, aparecían como atisbos los horizontes de lo otro. En el presente estudio, profundizaremos esta intencionalidad fundamental del exitente hacia la alteridad desde la perspectiva de la experiencia de la intersubjetividad, es decir, la experiencia de la relación entre subjetividades o personas. Para ello, comenzaremos primero preguntándonos con Marcel qué significa ser persona.

En una conferencia dictada en $1935^{3}$, Marcel profundiza sobre las nociones de acto y de persona, nociones que, a su parecer, han sido utilizadas inconsideradamente en los últimos años, sobre todo la noción de acto, que ha sido arrastrada a remolque de la idea de Revolución. Por ello, es necesario aproximarse concretamente al acto e intentar definirlo en algún grado-aún sabiendo que toda definición es imposible en el ámbito de la existencialidad. Por una parte, señala Marcel, es esencial al acto la capacidad de cambiar de algún modo una situación; por otra parte, el acto es determinado, tiene sus contornos que permiten distinguirlo como éste o aquél acto; además, la realidad del acto no se agota en la aparente conclusión del hacer, sino que "forma parte de la esencia del acto comprometer (engager) al agente» ${ }^{4}$. Esto último merece la atención especial de Marcel, y aclara que decir que mi acto me compromete significa que «lo propio de mi acto es el poder ser ulteriormente reivindicado por mí», es decir, que se sostiene en la afirmación ego sum qui feci: reconozco, pues, de antemano que, si me excusase de mi acto, me haría culpable de un reniego ${ }^{5}$. El ejemplo más claro de esta descripción de mi acto es el de la promesa, en la que «reconozco de antemano que si cuando se produzcan esas circunstancias [en las cuales he prometido mi asistencia], me excuso, me negaré a mí mismo, introduciré en mí una dualidad destructiva de mi propia realidad» ${ }^{6}$. De allí, pues, que no haya acto sin responsabilidad, razón por la cual postular un acto gratuito es contradictorio ${ }^{7}$. Retengamos la significación ontológica del acto, en tanto que el existente se define en su ser en el reconocimiento de la responsabilidad, por lo cual no se trata de una mera cuestión de contrato o de jurisdicción, sino que se trata de un reconocimiento o de un rechazo de la propia consistencia, la cual actúa y se realiza en sus actos ${ }^{8}$.

¿Todo esto nos proporciona los elementos de una definición del acto? En verdad no lo creo. Profundizando lo que acaba de ser dicho, se observará que forma parte de la esencia del acto no poder ser constatado o aprehendido objetivamente; no es pensable sin una referencia personal (référence personnelle), la referencia a un «soy yo quien...». Esto equivale a decir que el acto, por su parte, no se presenta como acto más que al agente o a quienquiera que abrace idealmente, por simpatía, la perspectiva del agente. Habrá, pues, que reconocer que pensar el acto no es ni puede ser objetivarlo, ya que al objetivarlo tiendo a tratarlo como no-acto (non-acte) ${ }^{9}$.

pp. 61-81. También: Grassi, M. «Existencia y encarnación en Gabriel Marcel», en: Contrastes. Revista Internacional de Filosofía, vol. XVI (2011), pp. 211-228.

3 «Remarques sur les notions d'acte et de personne», en: Essai de philosophie concrète. Paris: Gallimard, 1967 (en adelante: $R I$ ), pp. 159-180. Para las traducciones se ha seguido, en su mayor parte y cotejando con el original citado, la edición española: Filosofía Concreta. Madrid: Revista de Occidente, 1959.

$$
\begin{array}{ll}
4 & \text { RI, p. } 163 . \\
5 & \text { Ibid. } \\
6 & \text { Ibid. } \\
7 & \text { Ibid. } \\
8 & \text { Ibid. } \\
9 & \text { Ibid. }
\end{array}
$$


De ello resultan ciertas consecuencias importantes. Por un lado, nuestra propensión a objetivar es tal, que somos llevados a representarnos el acto como un efecto, y preguntarnos, por ello, de dónde sale o quién lo ha producido. Estamos obligados, pues, a reconocer un equívoco en el ego sum qui feci. En efecto, señala Marcel, damos una interpretación local a este «soy yo quien...», como si dijera que es esta mano o esta boca que tengo aquí la que ha actuado. Pero el valor significativo de esta localización es nulo, pues podría suceder que deba imputarme el acto o las palabras de otro. En este caso, yo no es cualquiera (quelqu'un), sino que alguien es algún otro: «Yo (moi) es de alguna manera lo contrario de cualquiera (quelqu'un), estoy aquí en un absoluto (je suis ici dans un absolu)» ${ }^{10}$. Claro que esto no representa más que un momento dialéctico, pues para los demás yo soy un alguien, un cualquiera, y mi acto es el de un alguien determinado; por otra parte, aprehendo a alguien cualquiera como agente, es decir, ver por sus ojos y verme como ellos me ven, como alguien, como algún otro. Así, ceso de coincidir conmigo, y es como si mi realidad se resquebrajase: tal es el resultado inevitable y falaz, para Marcel, de la introspección. En efecto, puedo considerar mi acto desde fuera, separarlo de mí y como si no fuese ya mío, como si fuese el acto de alguien. Así, el acto cesará de ser mi acto y, por tanto, cesará de ser un acto, transformándose en una especie de gesticulación: cuanto más el acto haya sido mi acto, es decir, cuanto más lo haya incorporado en la totalidad misma que yo soy, tanto menos podré considerarlo desde fuera. Se plantea, pues, una especie de jerarquía de los actos en cuanto tales: «un acto es tanto más acto cuanto menos posible me sea repudiarlo sin renegar íntegramente de mí mismo; y también esto hace aparecer la radical imposibilidad del acto gratuito ${ }^{11}$. El acto será tanto más acto cuanto más me comprometa como totalidad, como ser integral y consistente.

A partir de estas reflexiones en torno al acto, Marcel considera que podemos acercarnos a la noción de persona. El filósofo francés señala, en primer lugar, que no alcanzamos principalmente la esencia de la persona oponiéndola al individuo, ni tampoco oponiéndola a la cosa: «creo que es en su oposición al uno (man), al se (on) como se presenta la persona primeramente ${ }^{12}$. Este $s e$, si bien no es él mismo rigurosamente definible, se caracteriza, primero, porque es anónimo, sin rostro; en segundo lugar, es inasible, se escurre, "es por esencia irresponsable». En un sentido, es contrario de un agente. Su naturaleza es contradictoria, pues, se afirma como un absoluto, y es al mismo tiempo lo contrario de un absoluto. Marcel señala que nada hay más peligroso y difícil de evitar que el confundirlo con el pensamiento impersonal: el se es, en realidad, un pensamiento decaído, un no-pensamiento. La cuestión terrible estriba en que compruebo que el se está en el horizonte de mi conciencia y la oscurece, y me rodea por todas partes (sobre todo, agrega Marcel, en un mundo contaminado por la Prensa). Aún más, no sólo está alrededor mío, sino que me penetra, se expresa en mí y paso el tiempo reflejándolo: «mis opiniones, la mayoría del tiempo, no son otra cosa que la reproducción del se (on) por un yo (je), que ni siquiera sabe que lo reproduce» ${ }^{13}$. Ahora bien, hay que evitar la pseudoidea de que la persona se encontraría en un lugar entre ese se investidor y el se investido, cuestión que de por sí no tiene sentido. En realidad, lo propio de la persona, dice Marcel, es su posibilidad de afrontar (affronter): «desde este punto de vista diremos que el coraje (courage) es la virtud capital de la persona, mientras que el se aparece como

$\begin{array}{ll}{ }_{10} & \text { Ibid. } \\ 11 & \text { Ibid. } \\ 12 & \text { Ibid. } \\ 13 & \text { Ibid. }\end{array}$


el lugar mismo de toda fuga, de toda evasión». ${ }^{14}$ Ahora bien, la persona no solo afronta al se, sino que, por el hecho mismo de afrontarlo, lo rompe: al afrontarlo, lo obligo a salir, es decir, lo obligo a especificarse, a tomar un rostro. "En este sentido, la persona es la negación activa del se; yo no puedo reconocer el se, es decir, atribuirle aunque sólo fuese un rudimento de positividad, sin hacerme su cómplice, es decir, sin introducirlo en mí» ${ }^{15}$. Es preciso, indica Marcel, detectar un sentido intelectual al verbo afrontar, que significaría considerar. Ante todo, se considera una situación, y considerar una situación implica dejar de sufrirla sin más —es decir, dejar de aprehender al azar algunos de sus aspectos-, implica una especie de erección interior que se prolonga en el acto de afrontar. Considerar es, pues, afrontar. A su vez, considerar es evaluar, pues considerar una situación, de por sí indeterminada, es ya apreciarla y afrontarla, implica orientarse en un determinado sentido que permite fijarla y, por lo tanto, asumir la responsabilidad de mi orientación y del acto que surja de él ${ }^{16}$. Ahora bien, para Marcel, asumir es en un determinado sentido afrontar, pero ante todo significa afrontar el propio pasado, cosa extraña, pues se trata de afrontar algo que está detrás de sí. Así, si asumir es afrontar el propio pasado, se puede decir que afrontar una situación es asumirla como se asume la responsabilidad del propio acto, es decir, es tratarla como propia ${ }^{17}$.

Luego de estas aclaraciones, Marcel avanza sobre algunas perspectivas. En primer lugar, puede ahora reconocerse el verdadero sentido de la distinción entre individuo y persona ${ }^{18}$. En efecto, "el individuo es el se (on) en estado parcelario», es un elemento estadístico, "carece de mirada (regard), de rostro (visage)» ${ }^{19}$. En segundo lugar, las reflexiones anteriores posibilitan entrever lo que podría ser la persona absoluta (personne absolue), sin poder, empero, establecer aquí si se trata o no de una ficción metafísica: «para la persona absoluta, la ya precaria distinción entre el acto de afrontar y el de asumir queda totalmente abolida; tiende a presentarse íntegramente responsable de la historia $»^{20}$. Aún así, Marcel admite que no cree que de estos elementos pueda construirse una metafísica positiva, puesto que «la persona no es ni puede ser una esencia» ${ }^{21}$. En efecto, volviendo a la distinción entre individuo y persona, resulta imposible establecer una categoría metafísica de la persona, pues la persona no puede ser ni una variedad ni una elevación del individuo ${ }^{22}$.

La cuestión sobre el ser de la persona, pues, subsiste, y Marcel añade que «no podemos tratar a la persona ni como un dato, ni siquiera, quizá, como un existente» ${ }^{23}$. El problema es que Marcel no especifica por qué no podemos tratarlo como existente, algo extraño, teniendo en cuenta sus análisis de lo existencial. La persona no pueda ser tratada como un dato por la imposibilidad de objetivarla, o de pensarla como algo ya establecido, desconociendo que ante todo ser persona es algo a conquistar, es algo a lograr. Hasta ahora, la única fórmula que Marcel ofrece para dirigirse a la persona es la de que lo propio de la persona es afrontar. Pero, en el fondo, reconoce él mismo que dicha fórmula es deficiente en tanto que separa a la persona del acto en el cual se consuma.

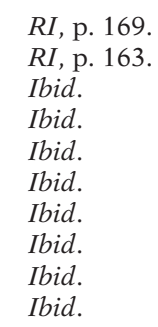


¿Cómo establecer una teoría de la persona que, al mismo tiempo, no la disocie de los actos personales? Podría pensarse que la persona es la unidad sintética que hace posible la conexión entre diversos actos, pero hay que notar que el problema que se pretende resolver es de orden teórico, es decir, surge para un espectador que convierte estos actos en determinaciones y los niega, por ello, en su especificidad. Decir que la persona es el sujeto de los actos, para Marcel, implica distanciarnos del acto en cuanto tal, representándonos el acto como operación y a la persona como operario. Prácticamente, señala Marcel, el deslizamiento hacia esta representación es absolutamente inevitable, puesto que estamos en lo parcelario, somos individuos, y estamos expuestos al se y, agrega, puede decirse que somos presa de la historia (sommes en proie à l'histoire). "Las contradicciones que he señalado — escribe Marcel— están, pues, en realidad, inscritas en nuestra condición misma; sin duda sólo por un esfuerzo de pensamiento torturante y casi imposible de prolongar llegamos a trascenderlas, de manera siempre precaria, además». ${ }^{24}$ Pueden extraerse de aquí dos conclusiones metafísicas opuestas, según Marcel: o bien la idea de persona humana es una ficción y, en su acepción más fuerte, sólo pueda afirmarse la persona de Dios; o bien, por el contrario, la persona permanece como correlato del elemento anónimo que afronta, por lo cual Dios no sería persona. Pero quizá, reconoce Marcel, se trate aquí más de una cuestión terminológica que real ${ }^{25}$.

Lo que complica la cuestión, por otra parte, es nuestra propensión a confundir persona y personalidad, por un lado, y acto y creación, por otra. En el primer caso, la personalidad se considera en tanto que signo o sello individual (Marcel recurre al alemán Prägung, que proviene de Das Prägen, palabra que significa acuñación, estampación, sello): «La personalidad en cuanto Prägung es innatismo; nos es dada, si no inmediatamente, al menos a través de medios tan misteriosamente transparentes como la voz o la mirada, en vez de que en los actos, en los que nos ha parecido ver que la persona se concentra, se diría que se ha hecho abstracción de todo innatismo, de todo enraizamiento ${ }^{26}$. Uno podría decir, dice Marcel, que la persona es la personalidad asumiéndose a sí misma, en tanto que pasado, es decir, el innatismo recibiendo de sí su propia consagración; sin embargo, no está seguro el filósofo de que esta solución sea inteligible o universalizable, puesto que hay casos en los que la persona llega a afirmarse a partir de un cierto "golpe de Estado» por el cual yugula lo que subsistía en ella de innatismo. En lo que respecta a la creación, sus conclusiones no son diferentes: «No hay seguramente creación fuera de un determinado misterio que envuelve al creador y brota a su través; de manera que lo que llamamos creación es en el fondo una mediación en el seno de la cual, como lo han visto los románticos [Schelling, particularmente: nota de 1966], pasividad y actividad se unen y se funden ${ }^{27}$. Así, se pregunta Marcel, ¿acaso la grandeza de la persona no reside en una determinada indigencia ontológica (indigence ontologique)? Los análisis de la persona, pues, terminan en preguntas, y Marcel no avanza más en este ensayo. Sin embargo, lo central aquí es subrayar la importancia de las palabras totalidad, exigencia, misterio y creación, pues sobre estas notas aparecerán las reflexiones en torno al tú y la esencial vocación de la persona a ser, o mejor, la posibilidad de entender la persona como vocación a ser.

\footnotetext{
$24 \quad R I$, p. 178.

25 RI, p. 163.

26 Ibid.

27 Ibid.
} 


\section{Yo y el Otro}

En una conferencia dictada en el Institut Supérieur de Pédagogie, en Lyon, en 1941, titulada Moi et autrui ${ }^{28}$, Marcel se detendrá en el análisis de lo que significa tanto el yo como el otro, y de qué modo se articulan estos términos en la existencia concreta. Comienza el ensayo afirmando que el yo es el resultado de un cierto acto de posición, acto que es siempre idéntico a sí mismo y que es preciso captar más allá de las ficciones que pudiera habernos legado la historia de la filosofía. Para ello, Marcel comienza por rastrear en la experiencia infantil una reivindicación del yo, puesto que el niño se afirma a sí mismo a partir de una determinada pretensión exclamativa («soy yo quien te ha traído estas flores»), y se afirma en su estar aquí presente frente a un otro del cual espera un reconocimiento de su propia individualidad y exclusividad. Este yo aqui presente no puede reducirse a ningún contenido especificable, sino que es una presencia global, que toma la figura de un centro de imantación. Pero la cuestión estriba en que esta pretensión de reconocimiento parece implicar una referencia al otro, pero se trata de un otro que funciona como la caja de resonancia de una complacencia consigo mismo. Lo interesante es señalar aquí que el yo no preexistía a esta relación al otro, en esta pretensión de reconocimiento, sino que, en todo caso, uno podría postular este yo preexistente y caracterizarlo por vía de la exclusión. En rigor, debe estudiarse el acto constitutivo del yo por el cual me represento al otro: «en el sentido etimológico de la palabra, yo me 'produzco' (produis), es decir, me pongo delante (je me mets en avant)» ${ }^{29}$. Así, el yo expresa una presencia que se insinúa en la experiencia, irreductible y confusa, que es el sentimiento de existir, de estar en el mundo ${ }^{30}$. Pero esta conciencia de existir se encuentra articulada a la pretensión de reconocimiento de sí por parte de un otro, por lo cual habrá que ver en el yo "no una realidad aislable, ya sea un elemento o un principio, sino un acento (accent) que confiero no a mi experiencia en su totalidad, sino a tal porción o a tal aspecto de esta experiencia que pretendo salvaguardar particularmente contra tal ataque o tal posible infracción ${ }^{31}$. De allí, señala Marcel, la imposibilidad de asignar a este yo fronteras precisas, puesto que se define en su referencia a un otro que puede ocupar posiciones siempre infinitamente variables.

Esta especie de enclave que es el yo está en constante movimiento y, más aún, en estado de vulnerabilidad. Esta condición existe a base de la angustia que despierta esta forma de saturación de sí mismo por la cual vigilo todo lo que procede del mundo exterior en el que me encuentro sumergido. Esta angustia o herida que yo soy es, ante todo, la experiencia de la contradicción entre el todo que aspiro a poseer y a monopolizar y la conciencia de la nada que soy, pues no puedo afirmar nada de mí mismo que sea auténticamente yo mismo, nada que sea permanente y se sustraiga de la crítica y de la duración: «de ahí esa necesidad loca de confirmación por lo exterior, por el otro, esa paradoja en virtud de la cual es del otro y sólo de él de donde, a fin de cuentas, el yo más centrado sobre sí mismo espera su investidura» ${ }^{32}$. Una experiencia concreta que parece revelar esta contradicción con fuerza innegable es la que se pone de relieve en la actitud de pose: el que adopta una pose parece estar preocupado sólo por los demás, cuando en realidad no se ocupa más que de sí mismo. Esta pretensión o complacencia en sí mismo

$\begin{array}{ll}28 & \text { Ibid. } \\ { }_{29} & \text { Ibid. } \\ { }_{30} & \text { Ibid. } \\ 31 & \text { Ibid. } \\ 32 & \text { Ibid. }\end{array}$


que está en la base de la pose atenta contra la autenticidad de todos los actos, palabras y actitudes personales. Pero no sólo el yo aquí se encuentra como negado en favor del otro, sino que el otro mismo, considerado como caja de resonancia o amplificador del sí mismo, se convierte en una especie de aparato del que dispongo y que puedo manipular: «más rigurosamente, se podría decir que el otro es el medium provisional, y como accesorio, a través del cual llego a formarme una cierta imagen, un cierto ídolo de mí mismo» ${ }^{33}$.

La cuestión del yo, pues, se desplaza desde una mera formulación teórica y abstracta -por ejemplo, si lo propusiéramos como principio superior del desarrollo personal, tal como aparece en una filosofía idealista-, hacia la reflexión en torno a las condiciones gracias a las cuales tomo conciencia de sí, condiciones que son esencialmente sociales. Marcel señala que vivimos en la actualidad en un régimen de competitividad y exasperación de la conciencia individual que lleva a un individualismo degenerante del sentido auténticamente comunitario del hombre. El sistema de examinaciones y oposiciones al que está sometido el hombre desde temprana edad incita a una permanente comparación respecto a los otros ${ }^{34}$. Tal régimen que exacerba la conciencia del yo o el amor propio es absolutamente despersonalizante, «pues lo que realmente tiene valor en nosotros es aquello que no es comparable, aquello que no tiene proporción (commune mesure) con otra cosa» ${ }^{35}$. El problema aquí, subraya Marcel, es que se ha impuesto una concepción que pretende encontrar en el yo el reducto o la guarida de la originalidad. Lo que se ignora y se desacredita en esta concepción es, en última instancia, la noción de don: «lo mejor de mí no me pertenece, no soy en absoluto su propietario, sino sólo su depositario ${ }^{36}$. Preguntar por el origen de los dones, por su procedencia, no tiene sentido - a no ser en un registro metafísico que es extraño al actual ${ }^{37}$. Lo que importa, por el contrario, es saber qué actitud adoptar frente a ellos: en última instancia soy llevado a considerarlos como la expresión de una llamada que me ha sido dirigida y a la que debo responder, por lo cual no tiene sentido enorgullecerme por ello o pavonearme frente a otros. En última instancia, insiste Marcel, «no hay nada en mí que no pueda o no deba ser considerado como don», y no es más que una ficción imaginar a un yo preexistente que tenga derecho o que merezca lo que le ha sido dado ${ }^{38}$.

Es preciso, señala Marcel, romper con esta idolatría falsa del yo, esta egolatría, y retomar la distinción entre el individuo y la persona. Lo propio de la persona consiste en afrontar directamente una situación dada y comprometerse efectivamente ${ }^{39}$. Pero no se trata aquí de identificar persona y yo, ni tampoco de concebir a la persona como una cosa distinta a esta otra cosa que es el yo, ni tampoco tomar a la persona como un elemento o un atributo del yo. Marcel escribe:

Mejor sería decir que [la persona] es una exigencia que ciertamente surge en lo que me aparece como siendo mío o como siendo yo, pero esta exigencia no toma conciencia de sí más que convirtiéndose en realidad: no puede, pues, de ninguna manera ser asimilada a una veleidad; digamos que pertenece al orden del "yo quiero», y no del «yo querría». Yo me afirmo como persona en la medida en que asumo la responsabilidad de lo que hago y de lo que digo. Pero cante quién soy o ante quién me reconozco

33 Ibid.

34 Ibid.

35 Ibid.

36 Ibid.

37 Ibid.

38 Ibid.

39 Ibid. 
responsable? Hay que responder que lo soy, al mismo tiempo, ante mí mismo y ante el otro, y que esta conjunción es precisamente característica del compromiso personal (engagement personnel), que es la marca propia de la persona ${ }^{40}$.

La persona, pues, es una exigencia ontológica, una exigencia de realización de lo propio, que no toma conciencia sino en su realización. Pero lo central aquí es que dicha realización implica una articulación con la alteridad, con los otros, en tanto que asumir la responsabilidad de mis realizaciones es responder de lo que soy ante mí y ante el otro, y esta doble respuesta se da en el compromiso personal, por lo cual la experiencia de la promesa y de la fidelidad tendrá un lugar capital en la reflexión en torno al ser de la persona ${ }^{41}$. La responsabilidad, pues, conlleva la afirmación del otro frente a quien soy responsable, por lo cual, afirma Marcel, me afirmo como persona en tanto que creo realmente en la existencia de los otros y en tanto mi conducta es deudora de esta creencia. Pero ¿qué significa aquí creer? «Es realizar o incluso afirmar esta existencia en sí misma, y no sólo en sus incidencias en relación a mí» ${ }^{42}$. Así, afirma Marcel, persona, compromiso, comunidad y realidad son nociones que no pueden desarticularse si es que queremos comprender la existencia personal ${ }^{43}$.

De este modo, llegamos a la noción tan central en Marcel de disponibilidad (disponibilité), la cual debe tomarse como característica esencial de la persona, y que arranca a la persona del estado de sonambulismo en la que se encuentra cuando se sumerge en el orden de lo objetivo, en el que se encuentra fascinado por los objetos a los que se refiere tanto el deseo como el temor. La disponibilidad corresponde al registro de la presencia, por lo cual no podemos entenderla como si significara una especie de vacío o de vacuidad que pide ser colmada o llenada. La disponibilidad «designa más bien una aptitud para darse a lo que se presenta y vincularse mediante este don; o incluso para transformar las circunstancias en ocasiones, digamos mismo en favores: a colaborar así con su propio destino confiriéndole su impronta propia ${ }^{44}$. Así, Marcel retoma la afirmación de que «la persona es vocación», y reconoce su verdad siempre y cuando se restituya a la palabra vocación su valor propio, que es el de ser una llamada o, aún mejor, una respuesta a una llamada, por lo cual dimana a la vez de mí mismo y de otra parte: en la vocación captamos «la más íntima conexión entre lo mío y lo que es de otro, conexión nutricia o constructiva que no puede aflojarse sin que el yo padezca anemia y se incline hacia la muerte» ${ }^{45}$.

Sólo la disponibilidad desactiva la mecanización o funcionalización tanto del otro como de mí mismo, para ganar la presencia personal de ambos, presencia en la que se encuentra la verdadera dignidad y valor de sí. El ser disponible se opone al ser que está ocupado o saturado de sí mismo, y más bien se encuentra tendido fuera de sí, consagrándose a una causa que lo sobrepasa pero que, al mismo tiempo, hace suya. Por ello, las ideas de creación, de potencia y de fidelidad creadora son las que se imponen aquí, afirma Marcel. Es preciso no confundir, sin embargo, creación y producción: «lo que es esencial en el creador es el acto por el que se pone a disposición (met à la disposition) de algo que, sin duda, en cierto sentido, depende de él para ser, pero que, al mismo tiempo, se presenta a él como más allá de lo que él es y de lo que puede juzgarse capaz de sacar directa e inmediatamente de sí» ${ }^{46}$. Marcel señala que esta caracterización bien puede

$\begin{array}{ll}40 & \text { Ibid. } \\ 41 & \text { Ibid. } \\ 42 & \text { Ibid. } \\ 43 & \text { Ibid. } \\ 44 & \text { Ibid. } \\ 45 & \text { Ibid. } \\ 46 & \text { Ibid. }\end{array}$


atribuirse al artista, a la misteriosa gestación que hace posible la aparición de la obra de arte. Y el proceso creador supone siempre, aunque no sea siempre manifiesto, una realización del progreso personal, en tanto que lo que una persona crea no es algo puramente exterior — como sucede en el caso de la producción-, sino que en la creación la persona se crea a sí misma. De allí que el filósofo pregunte: «¿Cómo no reconocer que la persona no se deja concebir fuera del acto por el cual ella se crea, pero al mismo tiempo que esta creación pende, en cierto modo, de un orden que la sobrepasa? » ${ }^{47}$.

De ser así, entonces, la persona no puede ser asimilada de ningún modo a un objeto, que está ahí, que está dado y presente ante nosotros, y que puede ser numerado o incluso agregado como elemento estadístico a un cálculo social. Desde el punto de vista, no ya externo a la personalidad, sino intrínseco a ella, parece imposible que la persona pueda afirmar de sí misma "yo soy»" ${ }^{48}$. La persona «se capta menos como ser que como voluntad de superar lo que en total es y no es, una actualidad en la que se siente, a decir verdad, comprometida o implicada, pero que no la satisface: que no está a la altura de la aspiración con la que se identifica ${ }^{49}$. Por ello, Marcel afirma que el lema de la persona no es sum (soy), sino sursum (seré). Sin embargo, Marcel advierte del peligro de un cierto romanticismo común a todas las épocas por el cual se desestima cualquier realización en pos de un posible que quizá nunca se realice. En el caso de la persona no se trata de ese tipo de aspiración - más propia del yo y del deseo. Hay en la persona una exigencia de encarnación, que significa que la persona se realiza sólo en el acto por el que tiende a encarnarse (en un acto, una obra, el conjunto de su vida), aunque le sea esencial, al mismo tiempo, no paralizarse o cristalizarse en ninguno de ellos, puesto que participa de la plenitud inagotable del ser del que emana toda su dinámica. "Ahí está la razón profunda por la cual es imposible pensar la persona o el orden personal sin considerar al mismo tiempo lo que está más allá de ella o de él, una realidad suprapersonal que preside todas sus iniciativas, que es a la vez su principio y su fin» ${ }^{50}$. La persona, pues, no puede entenderse sino desde las coordenadas de la exigencia ontológica, la vocación ontológica - podríamos decir-, que se manifiesta en una permanente recreación de sí en su respuesta libre a las llamadas que provienen de lo profundo de sí y que, por lo demás, se revelan gracias a los otros ${ }^{51}$.

\section{El tú COMO PRESENCIA}

La persona se encuentra en un plano ontológico radicalmente distinto al del objeto ${ }^{52}$. Lo personal comparte con la existencia la inmediatez de su realidad, es decir, la imposibilidad de establecerse en la indiferencia para referirse a ellas. Esta imposibilidad de tomar distancia y hacer las veces de un espectador frente a la existencia y a lo personal se expresa, en Marcel, gracias a la noción de presencia. La presencia, por impugnar el divorcio entre un sujeto y un objeto, escapa al ámbito de lo problemático para jugarse en el plano de lo meta-problemático o misterioso, plano en el que encontramos la

$\begin{array}{ll}{ }_{47} & \text { Ibid. } \\ { }_{48} & \text { Ibid. } \\ 49 & \text { Ibid. } \\ { }_{50} & \text { Ibid. } \\ 51 & \text { Ibid. } \\ 52 & \text { Ibid. }\end{array}$


participación y la comunión ${ }^{53}$. Sólo con un ser presente podemos comunicarnos, en el sentido de estar con..., de sentirnos presentes junto a un ser igualmente presente a nosotros y en cuyo encuentro ambos se realizan, adquieren realidad y consistencia ${ }^{54}$.

Antes de avanzar, debemos especificar una de las distinciones fundamentales de la filosofía marceliana: la de problema y misterio. Mientras que lo problemático es lo propio del ámbito de la objetividad, lo misterioso se presenta en el ámbito de la existencia o del ser ${ }^{55}$, en el ámbito ontológico ${ }^{56}$. Lo problemático, en efecto, supone la separación y la insularidad que supone el ser puesto frente a mí propio del objeto. El misterio, en cambio, es el reconocimiento de una unidad de la experiencia personal que no puede cercenarse, que es la unidad pletórica de lo existencial, en donde la separación de sujeto y objeto pierde sentido, y en el seno de la cual me encuentro implicado ${ }^{57}$. Para Marcel, el mejor modo de acceder al sentido y significado del misterio es el de «mostrar la diferencia de registro espiritual que separa al objeto de la presencia ${ }^{58}$. Podemos tomar como ejemplo concreto el hecho de sentir a alguien que está a nuestro lado en una misma habitación, a quien podemos ver, oír y tocar, y que, sin embargo, no está presente; aún más, que se encuentra infinitamente más lejos nuestro que una persona a quien amamos y que, sin embargo, se encuentra a miles de kilómetros de nuestra ubicación, o aún más, que ya no se encuentra en este mundo. Claro que en el primer caso hay una especie de comunicación, en tanto que el individuo que está a mi lado puede también oírme, verme, tocarme: pero se trata sólo de una comunicación material, semejante a la que puede establecerse entre dos elementos, uno emisor y otro receptor. La cuestión estriba en que falta aquí el elemento esencial.

Podría decirse que es una comunicación sin comunión (communication sans communion), y que por eso es una comunicación irreal. El otro oye mis palabras, pero no me oye a mí mismo; hasta puedo tener la impresión extrañamente penosa que estas palabras, tal como me las devuelve o refleja, son para mí irreconocibles. Por un fenómeno singular, el otro se interpone entre mi propia realidad y yo; me vuelvo extraño a mí mismo; no soy yo mismo cuando estoy con él. Pero, por un fenómeno inverso, puede ocurrir que el otro, cuando lo siento presente, en cierto modo me renueve interiormente; esta presencia es entonces reveladora, es decir, me hace ser plenamente lo que yo no sería sin ella ${ }^{59}$.

\footnotetext{
53 Ibid.

$54 \quad$ Ibid.

55 Ibid.

$56 \quad$ Ibid.
}

57 Cf. EA, p. 145. «In one sense the idea of mystery, or at least the term mystery, is simply one of several inadequate ways to point toward the character and fundamental reality of personal experience. It is not especially more or less important than several other ideas and terms that serve the same purpose. In another sense all of Marcel's philosophical research attempts to penetrate and evoke mystery. In this sense there is no idea of mystery in the philosophy of Marcel because his idea of mystery and his philosophy are the same. But mystery here is not an idea - that is, an abstraction- so much as it is the central character and being of the concrete experiences Marcel seeks to explore» (ReEves, G. «The idea of mystery in the philosophy of Gabriel Marcel», en: AA.VV. The Philosophy of Gabriel Marcel. The Library of Living Philosophers, vol. XVIII, ed. Schilpp \& Hahn, Carbondale: Southern Illinois University, 1984, pp. 246247). En la carta en respuesta a este artículo, contenido en el mismo volumen a continuación, Marcel escribe: "Consequently, the words mystery of being translate in a very schematic and therefore inevitably inadequate way the fact that as a thinking being I am involved in a vast communion, a vast co-esse, of which I can have, apart from a metaphysical assertion, only a fragmentary awareness through key experiences, to which endeed I must always direct myself if I do not want to bog down in general, sterile affirmation» (p. 274).

$58 R I$, p. 163.

59 Ibid. 
Aún cuando estas experiencias presenten un carácter irrecusable, no se dejan traducir en un lenguaje discursivo, puesto que, mientras el objeto está sujeto a un conjunto de técnicas enseñables y, por ello, transmisibles, la presencia escapa absolutamente a la transmisión. La comunión, propia de la presencia, se opone a la transmisión, propia del objeto, y no se sitúan en la misma región del ser; aún más, «toda transmisión se efectúa —si se me permite la expresión- más aquí del ser (en deça de l'être)» ${ }^{60}$. El problema se encuentra cuando intentamos pensar la presencia desde las coordenadas del objeto: en rigor, el acto por el cual nos orientamos a la presencia es esencialmente distinto de aquél gracias al cual aprehendemos el objeto ${ }^{61}$. Aún más, en el ámbito de la presencia es inexacto hablar de captación o aprehensión, sino que «la presencia únicamente puede acogerse (accueillie) (o rechazarse)» ${ }^{62}$. La distancia entre captar y acoger es abismal, la actitud fundamental es radicalmente distinta: no podemos acoger o aceptar un objeto, sino que el objeto está allí para ser tomado o dejado donde se encontraba, y aquí tomar indica también el acto de la inteligencia por el cual se comprende algo. Así, en tanto que la presencia se sustrae a la aprehensión, también se encuentra fuera de las posibilidades de la comprensión: «la presencia sólo puede invocarse o evocarse, y la evocación es, en suma, de esencia mágica ${ }^{63}$. Por esta razón, la presencia sólo es percibida de manera intermitente (which can only be glapsed at, afirma Marcel utilizando una expresión inglesa).

Es interesante notar que las reflexiones en torno a la intersubjetividad han brotado en Marcel no desde exigencias sistemáticas de una filosofía, sino de experiencias de vida muy concretas. Por un lado, en su vocación dramatúrgica, Marcel pone especial acento en el encuentro entre personas reales de carne y hueso, y en la búsqueda de sentido que se emprende siempre junto con un otro $^{64}$. Por otro lado, la reflexión en torno a la intersubjetividad se nutre de la experiencia de la muerte del ser amado y el enigma de su presencia ${ }^{65}$. Por último, y especialmente, hay que subrayar la importancia decisiva que marcaron en la marcha filosófica de Marcel las experiencias con los fenómenos para-normales o para-psicológicos. Aún con las críticas que él mismo esgrimiera respecto a ciertas prácticas espiritistas, estas experiencias para-psicológicas o meta-psíquicas despertaron en el filósofo francés la tarea de pensar la presencia más allá de las coordenadas del objeto. Como él mismo remarca, estas experiencias han sido en general desestimadas por los filósofos, y han sido privadas de su riqueza ontológica, pero en él han jugado un papel esencial para adentrarse en el misterio de la intersubjetividad ${ }^{66}$. Para Marcel, estamos tan habituados a la naturalidad de lo dado, a su inscripción en el registro de la explicación causal, que perdemos de vista la extrañeza fundamental de lo dado, la extrañeza a la que, en cambio, nos enfrentan experiencias tales como las de la telepatía ${ }^{67}$. En efecto, es en el marco de la reflexión en torno a la telepatía en la que Marcel se detiene en el significado de la palabra «con» y a la realidad de la comunidad intersubjetiva, tal como aparecen en su Journal Métaphysique. Recordemos que es a partir de su experiencia en la Cruz Roja como Marcel se acerca a las experiencias espiritistas, intentando rescatar la presencia de aquellas personas perdidas en la guerra, que eran

$\begin{array}{ll}60 & \text { Ibid. } \\ 61 & \text { Ibid. } \\ 62 & \text { Ibid. } \\ 63 & \text { Ibid. } \\ 64 & \text { Ibid. } \\ 65 & \text { Ibid. } \\ 66 & \text { Ibid. } \\ 67 & \text { Ibid. }\end{array}$


completamente objetivadas y registradas en el régimen de búsqueda y de información de los organismos burocráticos del Estado ${ }^{68}$.

Nuestra lógica es, a los ojos de Marcel, absolutamente insuficiente y pobre para reflexionar en torno a las experiencias que se designan con la palabra con (avec): como ya habíamos señalado, no basta con estar cerca de una persona en un lugar para estar con ella: «la palabra con no tiene sentido más que allí donde hay una unidad sentida (unité sentie)» ${ }^{69}$. Las coordenadas de tiempo, lugar y causalidad, gracias a las cuales la experiencia se articula lógicamente, quedan relegadas frente a esta experiencia del encuentro. En todo caso, el encuentro, que se realiza en la comunidad entre el yo y el tú, sólo puede pensarse desde la invocación, pero en tanto que la invocación se distingue esencialmente de la representación, es decir, del conocimiento de lo otro por la idea. Y hasta tal punto deben distinguirse ambas operaciones que, en rigor, sólo es posible la eficacia de la invocación en tanto que la comunidad es ya una realidad, en tanto que el yo y el tú están ya juntos $^{70}$. Justamente, es gracias a esta comunidad que la invocación puede tener lugar, pues invocamos tan sólo a quien puede respondernos, es decir, al reconocimiento de un tú con quien comulgamos ${ }^{71}$. A su vez, este reconocimiento del tú sólo será posible si abandonamos nuestras expectativas y presunciones respecto a la persona que tenemos delante y con quien comulgamos, por lo cual es la humildad y la disponibilidad las que aparecerán como actitudes fundamentales para la comunión y el reconocimiento, y quizá sea el modo de encontrar un camino hacia el reconocimiento de un Tú Absoluto, quien se encuentra con nosotros sin ningún tipo de pretensión (más propia del egoísmo) ${ }^{72}$. Sólo en tanto que nos mantengamos disponibles a la revelación de otro podemos efectivamente comulgar con él, es decir, en tanto que rompamos con el círculo que formamos con nosotros mismos y que se materializa en la idea que representa al otro ${ }^{73}$.

Para Marcel es fundamental distinguir al tú del él: tratar al otro como a un tú no es asimilable a un mayor conocimiento de la esencia objetiva del otro, sino que, por el contrario, tratar al otro como un tú implica tratarlo como libertad, y ya no como naturaleza, es decir, implica promocionar su libertad y ayudarle a ser libre — tal como revela la experiencia del amor-, respetando y reconociendo su alteridad en su libertad (si fuera el otro solo naturaleza, no se distinguiría de uno mismo, que comparte dicha esencia natural). ${ }^{74}$ De aquí, también, que la cuestión de la existencia del otro, del tú, sea

68 Ibid.

69 Ibid.

70 Ibid.

71 Ibid.

72 Ibid.

73 Ibid.

74 «Lorsque je traite un autre comme un toi et non plus comme un lui, cette différence de traitement ne qualifie-t-elle que moi-même, mon attitude envers cet autre, ou bien puis-je dire qu'en le traitant comme un toi je pénètre plus avant en lui, que j’appréhende plus directement son être ou son essence? Ici encore il faut prendre garde; si par «pénétrer plus avant», ou par «appréhender plus directement son essence» on veut dire parvenir à une connaissance plus exacte ou en un sens quelconque plus objective il faut sans aucun doute répondre non. A cet égard, il sera toujours possible, si l'on s'en tient à un mode de détermination objective, de dire que le toi est une illusion. Mais remarquons que le terme même d'essence est extrêmement ambigu; par essence on peut entendre ou une nature ou une liberté; il est peut-être de mon essence en tant que liberté de pouvoir me conformer ou non à mon essence en tant que nature. Il est peut-être de mon essence de pouvoir n'être pas ce que je suis; tout simplement de pouvoir me trahir. Ce n'est pas l'essence en tant que nature que j'atteins dans le toi. En effet en le traitant comme lui je réduis à l'autre à n'être que nature: un objet animé qui fonctionne de telle façon et non de telle autre. Au contraire, en traitant l'autre comme toi, je le traite, je le saisis comme liberté; je le saisis comme liberté, car il est aussi liberté et non pas seulement nature. Bien plus je l'aide en quelque sorte à être libéré, je collabore à 
problemática, en tanto que pareciera que el hecho de ser un sí mismo no puede hacer las veces de un predicado, y este modo substancialista de comprender al otro parece sostenerse en una metafísica del él, ignorando la presencia misteriosa del tú: por ello, afirma Marcel, «el tú es a la invocación lo que el objeto es al juicio» ${ }^{75}$. Claro que, por otro lado, si tenemos en cuenta la distinción entre existencia y objetividad, y entendemos a la existencia como presencia, entonces debemos decir que la existencia no puede atribuirse más que a los otros, y a mí mismo en tanto que no soy esos otros ${ }^{76}$. La dificultad se encuentra en que, como hombres, somos a la vez objetos del mundo y presencias libres, y en esta ambigüedad se encuentra el corazón del drama del reconocimiento, puesto que soy tanto un él como un tú para los otros y para mí mismo. En todo caso, la intersubjetividad y la presencia no pueden ser escindidas.

[L]a presencia es intersubjetiva (la présence est intersubjective), no puede interpretarse más que como expresión de una voluntad que busca revelarse a mí. Pero esta revelación supone que yo no la obstaculizo; en suma, el sujeto es tratado no como un objeto, sino como foco (foyer) de imantación de la presencia. En la raíz de la presencia hay un ser que me tiene en cuenta (qui tient compte de moi), que lo pienso como teniéndome en cuenta; de allí que, por definición, el objeto no me tiene en cuenta, que yo no soy para él' ${ }^{77}$.

No podemos pensar la intersubjetividad más que si el otro me tiene en cuenta, así como yo lo tengo en cuenta a él: el encuentro y la comunión sólo son posibles en tanto que rompemos la cerrazón de sí sobre sí y atendemos a la presencia de otro ${ }^{78}$. Por esta razón, es insuficiente e inexacto decir que la intersubjetividad es una relación, puesto que la relación se da entre dos términos exteriores uno al otro. Por el contrario, la presencia parece venirme desde dentro, desde el interior, por lo cual la presencia es misteriosa (recordemos que lo propio de lo problemático y lo objetivo es su carácter de exterioridad) y se mantiene desatendida si la abordamos desde fuera, como si la consideráramos susceptible de responder a una encuesta que le dirijo, al modo del sociólogo o del psicólogo ${ }^{79}$. La intersubjetividad, pues, expresa la comunión y el encuentro entre presencias libres e irreductibles a la condición de cosa, por lo cual, como en todo ámbito metafísico, se coloca en el registro de lo meta-problemático o misterioso ${ }^{80}$. Por otro lado, la misma noción de persona cambia radicalmente de signo al entenderse como apertura a un $t u$, es decir, al dejar de ser considerada desde los caracteres de la identidad y la inmanencia, para comprenderse como encerrando en su núcleo la alteridad y la trascendencia ${ }^{81}$.

Universidad Católica Argentina

MarTín Grassi

Academia Nacional de Ciencias de Buenos Aires - CONICET

martingrassi83@gmail.com

[Artículo aprobado para publicación en diciembre de 2015]

sa liberté - formule qui paraît extrêmement paradoxale et contradictoire, mais que l'amour ne cesse de vérifier. Mais d'autre part c'est en tant que liberté qu'il est véritablement autre; en tant que nature en effet il m'apparaît identique à ce que je suis moi-même en tant que nature - et c'est sans doute par ce biais et par ce biais seulement que je puis opérer sur lui par suggestion (confusion redoutable et fréquent entre l'efficacité de l'amour et celle de la suggestion)»(EA, pp. 153-155).

$75 \quad R I$, p. 163.

76 Ibid.

77 Ibid.

78 Ibid.

79 Ibid.

80 Ibid.

81 Ibid. 